\title{
Electron-electron interactions in graphene field-induced quantum dots in a high magnetic field
}

Orlof, A.; Shylau, Artsem; Zozoulenko, I. V.

\section{Published in:}

Physical Review B

Link to article, DOI:

10.1103/physrevb.92.075431

Publication date:

2015

Document Version

Publisher's PDF, also known as Version of record

Link back to DTU Orbit

Citation (APA):

Orlof, A., Shylau, A., \& Zozoulenko, I. V. (2015). Electron-electron interactions in graphene field-induced quantum dots in a high magnetic field. Physical Review B, 92(7), [075431].

https://doi.org/10.1103/physrevb.92.075431

\section{General rights}

Copyright and moral rights for the publications made accessible in the public portal are retained by the authors and/or other copyright owners and it is a condition of accessing publications that users recognise and abide by the legal requirements associated with these rights.

- Users may download and print one copy of any publication from the public portal for the purpose of private study or research.

- You may not further distribute the material or use it for any profit-making activity or commercial gain

- You may freely distribute the URL identifying the publication in the public portal

If you believe that this document breaches copyright please contact us providing details, and we will remove access to the work immediately and investigate your claim 


\title{
Electron-electron interactions in graphene field-induced quantum dots in a high magnetic field
}

\author{
A. Orlof* \\ Mathematics and Applied Mathematics, MAI, Linköping University, SE-581 83 Linköping, Sweden \\ A. A. Shylau ${ }^{\dagger}$ \\ Center for Nanostructured Graphene (CNG), Department of Micro- and Nanotechnology, \\ DTU Nanotech, Technical University of Denmark, DK-2800 Kongens Lyngby, Denmark \\ I. V. Zozoulenko \\ Laboratory of Organic Electronics, ITN, Linköping University, SE-601 74 Norrköping, Sweden \\ (Received 25 March 2015; revised manuscript received 1 July 2015; published 20 August 2015)
}

\begin{abstract}
We study the effect of electron-electron interaction in graphene quantum dots defined by an external electrostatic potential and a high magnetic field. To account for the electron-electron interaction, we use the Thomas-Fermi approximation and find that electron screening causes the formation of compressible strips in the potential profile and the electron density. We numerically solve the Dirac equations describing the electron dynamics in quantum dots, and we demonstrate that compressible strips lead to the appearance of plateaus in the electron energies as a function of the magnetic field. Finally, we discuss how our predictions can be observed using the Kelvin probe force microscope measurements.
\end{abstract}

DOI: 10.1103/PhysRevB.92.075431

PACS number(s): 73.22.Pr, 73.21.La

\section{INTRODUCTION}

Semiconductor heterostructure quantum dots have been a topic of numerous experimental and theoretical studies $[1,2]$. Many of the electronic properties of quantum dots are by now well understood, and an application of quantum dots in optoelectronic devices (solar cells, light-emitting diodes), single-electron devices (transistors), and spintronics in quantum computing (qubits) is being explored [3]. With the discovery of graphene in 2004 [4], there was a new and interesting material for quantum dot fabrication. Graphene has several important features that make it different from conventional semiconductors: (i) its low-energy electrons behave like massless particles, (ii) its spectrum is gapless, and (iii) its edge/boundary can have a drastic effect on its electronic properties. To base the electronic devices mentioned above on quantum dots made of graphene instead of a semiconductor material, one needs to investigate how the basic properties of quantum dots depend on their composite material.

There are several types of graphene quantum dots, including islands, i.e., dots cut from graphene and defined by their edge geometry [5-11], and field-induced dots, which are defined by the application of electric and magnetic fields [5,12,13]. In this paper, we focus on the latter as they are experimentally feasible and susceptible to changes caused by a variation of the electrostatic potential and the magnetic field [14]. As electrons in graphene behave like massless particles, they undergo Klein tunneling [15], and the electrostatic confinement that is possible in normal semiconductors becomes impossible for graphene. Consequently, one cannot construct a graphene quantum dot using the electrostatic potential only. The spatial confinement of electrons can be achieved by an additional

\footnotetext{
*anna.orlof@liu.se

†arts@nanotech.dtu.dk

†igor.zozoulenko@liu.se
}

application of the magnetic field, which when high enough [16] or properly tuned, leads to electron localization [12,17]. The electrostatic potential creates a quantum well, but even after the addition of the magnetic field, there can be states with the oscillatory amplitude outside the barrier. With the increase in the magnetic field, such states gain an exponentially decaying amplitude, which is needed for the confinement [17].

There are a number of publications investigating the properties of such field-induced quantum dots [12,17-22]. The theoretical research presented in these publications focuses on the analysis of electrons dynamics (eigenstates and eigenenergies of the Dirac equation) in different types of confining circularly symmetric potentials in a perpendicular magnetic field. The potentials considered were piecewise rectangular quantum wells or smooth radially symmetric potentials described, for example, by a power function [12] or a Gaussian [18]. Even though there are no bound states in graphene quantum dots defined by the electrostatic potential only, quasibound states are possible, in particular, in sharp boundary wells when electrons have energies close to the barrier height [23]. Moreover, the presence of the magnetic field does not always lead to confinement. For example, for the case of the Gaussian potential in a low magnetic field, electrons can escape into the potential barrier, which is manifested in the anticrossing in the energy spectrum [17]. On the other hand, for a smooth circularly symmetric electrostatic potential described by the power functions, the confinement depends on the interplay between the electric and magnetic fields. Hence, for the low perpendicular magnetic field described by a circular symmetric azimuthal vector potential given by a power-law function, one can compare the electrostatic and magnetic-field profiles. Here, the confinement is present for the slope of a magnetic vector potential that is steeper than the electrostatic potential profile; when the slopes are equal, a confinementdeconfinement transition occurs, also manifested in the energy anticrossings [12]. When the electrostatic potential is steeper than the magnetic vector potential, the states are unbound and 
oscillate. Such states are present in a parabolic electrostatic potential with a linear magnetic vector potential, however here resonant quasibound states are possible in the low-field regime, whereas in the high-field regime the Landau levels are recovered [19].

All of the previous studies used smooth model potentials (power-law or Gaussian) to calculate the energy spectrum of graphene quantum dots. At the same time, it is known that in conventional semiconductor heterostructures, the electronelectron interaction in a high magnetic field can strongly modify the potential, leading to the formation of compressible strips [24]. These compressible strips are known to affect and alter the electronic properties of quantum dots and antidots [25-27]. Even though certain aspects of electron-electron interactions have been considered previously [28,29], the effect of magnetic-field-induced modification of the confining potential on the eigenspectrum of graphene quantum dots has not been studied before, and the present paper represents a step in this direction.

Another motivation for our study is related to the possibility to investigate the properties of quantum dots and antidots in the confined geometry in the regime of edge-state transport. Numerous studies of such structures in conventional (mostly GaAs) semiconductor heterostructures have revealed a variety of interesting phenomena, such as a new form of the Coulomb blockade seen in electrostatically confined dots and charging in open systems [30], unexpected periodicity of the AharonovBohm oscillations [31], just to mention a few examples. The formation and charging of the compressible strips in the structures at hand are believed to be responsible for many of the observed phenomena [32,33], and therefore a detailed investigation of the character of the confining potential and the structure of the energy levels can be important for designing and understanding the transport experiments in the confined geometry for graphene quantum dots and antidots.

In this paper, we study the effect of the electron-electron interaction on the level of the Hartree approximation (neglecting exchange, correlations, and spin). To outline the role of the electron-electron interaction in the presence of magnetic field $B$, we use the Thomas-Fermi approach and perform two different calculations for the self-consistent total potential. Here it is important to mention that the Thomas-Fermi approximation corresponds well with the quantum-mechanical methods for the calculation of electron density and electrostatic potential in semiconductor as well as graphene systems [34,35].

First, we set $B=0$, and for a given external applied electrostatic potential we calculate self-consistently the resulting total potential $V_{\text {mod }}(r)$ (which we will refer to as a model potential). It has a smooth shape similar to those of the model potentials studied previously [12,17-22]. Then we turn on the magnetic field and perform similar self-consistent calculations for the total potential $V(r)$ for the case $B \neq 0$. Using the obtained potentials in the Dirac Hamiltonian, we calculate the corresponding eigenenergies and thus investigate the effect of the electron-electron interaction on the electronic properties of a quantum dot in a high magnetic field.

The paper is organized as follows. In Sec. II, we present the Dirac model for graphene field-induced quantum dots. The model includes electron-electron interaction on the Hartree level within the Thomas-Fermi approximation via the screened potential calculated self-consistently (Sec. II B). To investigate the effect of the electron-electron interaction in the quantum dot, we solve the Dirac equation numerically (Sec. IIC) and analyze the electron eigenenergies (Sec. III). Finally, we suggest and discuss the possible experimental testing of our predictions (Sec. IV), and we conclude the article in Sec. V.

\section{MODEL AND THE SOLUTION METHOD}

\section{A. Basics}

We consider a system shown in Fig. 1. It consists of a graphene sheet on an insulating substrate with a dielectric constant $\epsilon_{r}=3.9$ (corresponding to $\mathrm{SiO}_{2}$ ) of a thickness $d$. A charge density of the graphene surface is controlled by the gate voltage $V_{g}$ applied between a metallic back gate and graphene. Applying an external potential $V_{\text {ext }}$ and a magnetic field $B$ perpendicular to the graphene layer, we confine electrons in the middle of the sheet creating a quantum dot. The external potential can be generated by a top gate electrode or a scanning tunneling microscope tip $[12,17]$.

The electron dynamics in the vicinity of the Dirac point in graphene is described by the Dirac Hamiltonian [36],

$$
H=\hbar v_{F} \sigma(\mathbf{p}-e \mathbf{A})+V(r)
$$

where $\mathbf{p}=-i \hbar \nabla$ is the momentum, $v_{F} \approx 10^{6} \mathrm{~m} / \mathrm{s}$ is the Fermi velocity, $e$ is the electron charge, $\sigma$ are the Pauli matrices, $\mathbf{A}$ is the magnetic vector potential, and $V(r)$ is the total screened potential, which is assumed here to be circularly symmetric.
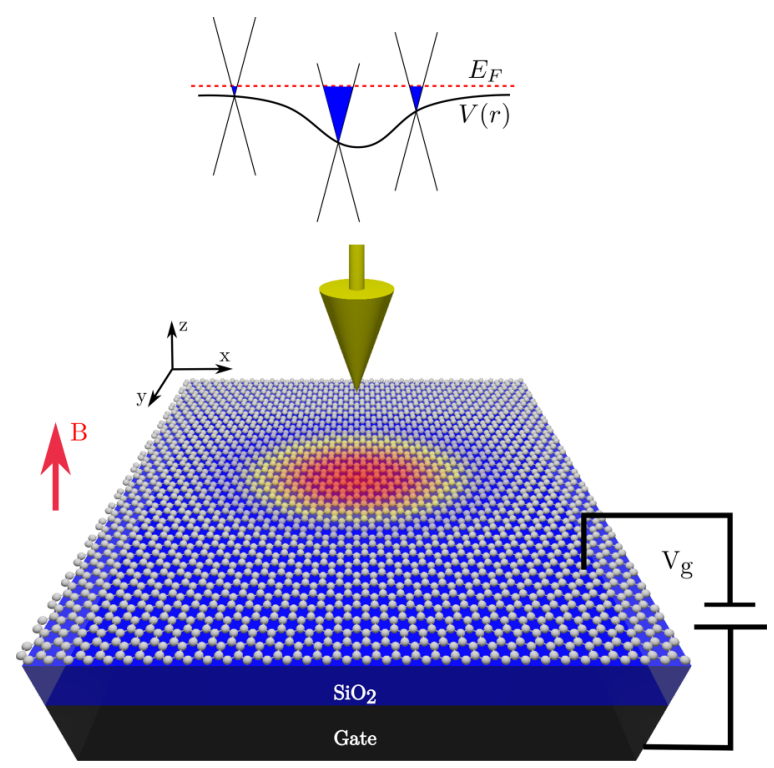

FIG. 1. (Color online) Sketch of a system under consideration representing a graphene sheet on an insulating substrate $\mathrm{SiO}_{2}$ (blue) and a back gate (black). A quantum dot is created via an application of electrostatic potential (red) from a tip and a perpendicular magnetic field $B$. A gate voltage $V_{g}$ is applied to the back gate. In the sketch of the profile of the quantum dot $[V(r)]$ and the Fermi energy $\left(E_{F}\right)$, we illustrate the Thomas-Fermi approximation, where the position of the Dirac point depends on $r$, and the density of states is calculated locally. 


\section{B. Potential and electron-electron interaction}

We treat the electron-electron interaction within the Thomas-Fermi approximation and include it in the Hamiltonian via a screened potential $V(r)$ calculated self-consistently from the external applied potential $V_{\text {ext }}$ using the mirror charge method. The procedure of finding the screened potential is as follows:

First of all, the Thomas-Fermi approximation (Fig. 1) assumes a local relation between the charge density $n$ and the total potential $V(r)$ [37-39],

$n(r)= \begin{cases}-\int_{V(r)}^{\infty} \rho^{e}[E-V(r)] f_{\mathrm{FD}}^{e}\left(E, E_{F}\right) d E & \text { for electrons, } \\ \int_{-\infty}^{V(r)} \rho^{h}[E-V(r)] f_{\mathrm{FD}}^{h}\left(E, E_{F}\right) d E & \text { for holes, }\end{cases}$

with the Fermi-Dirac (FD) distribution function

$$
f_{\mathrm{FD}}^{e / h}\left(E, E_{F}\right)=\frac{1}{\exp \left( \pm \frac{E-E_{F}}{k_{B} T}\right)+1},
$$

and the density of states (DOS) [36]:

$$
\rho^{e / h}(E)=\sum_{N=0}^{\infty} \frac{g}{2 \pi l_{B}^{2}} \delta\left(E \mp \hbar \omega_{c} \sqrt{n}\right),
$$

where a factor $g$ describes a spin and valley degeneracy with $g=4$ for all the levels but the zeroth one where we set $g=2$ as the level is shared between electrons and holes; $E_{F}=e V_{g}$ is the Fermi energy, $k_{B}$ is the Boltzmann constant, $\omega_{c}=\frac{\sqrt{2} v_{F}}{l_{B}}$ is the cyclotron frequency, and $l_{B}=\sqrt{\frac{\hbar}{e B}}$ is the magnetic length.

The total potential is a sum of the external potential and the Hartree term,

$$
V(r)=V_{\text {ext }}(r)+V_{\text {Hartree }}(r) .
$$

The external potential describes the effect of the top gate (or a STM tip) and is assumed to be of the Gaussian form

$$
V_{\text {ext }}(r)=e V_{0} e^{-\frac{1}{2}\left(\frac{r}{l_{0}}\right)^{2}},
$$

where $V_{0}$ and $l_{0}$ define a magnitude and an extension of the potential, respectively. The Hartree potential is defined in a standard way,

$$
V_{\text {Hartree }}(r)=\frac{e^{2}}{4 \pi \epsilon_{0} \epsilon_{r}} \iint\left(\frac{n\left(r^{\prime}\right)}{\left|\mathbf{r}-\mathbf{r}^{\prime}\right|}-\frac{n\left(r^{\prime}\right)}{\sqrt{\left|\mathbf{r}-\mathbf{r}^{\prime}\right|^{2}+4 d^{2}}}\right) d S^{\prime},
$$

where integration is performed over the graphene sheet, and the last term gives a contribution from the mirror charges. Because of a radial symmetry of the system at hand, we can integrate over the angle and obtain

$$
\begin{aligned}
V_{\text {Hartree }}(r)= & \frac{e^{2}}{\pi \epsilon_{0} \epsilon_{r}} \int_{0}^{\infty} n\left(r^{\prime}\right) \\
& \times\left[\frac{K\left(\frac{2 \sqrt{r r^{\prime}}}{r+r^{\prime}}\right)}{r+r^{\prime}}-\frac{K\left(2 \sqrt{\frac{r r^{\prime}}{4 d^{2}+\left(r+r^{\prime}\right)^{2}}}\right)}{\sqrt{4 d^{2}+\left(r+r^{\prime}\right)^{2}}}\right] r^{\prime} d r^{\prime},
\end{aligned}
$$

where $K(r)$ is the elliptic integral of the first kind. Then using Eqs. (2)-(9) we can calculate self-consistently the total potential $V(r)$, which accounts for both screening and applied magnetic field. To outline the effect of the magnetic field in the total self-consistent potential, we will compare $V(r)$ with a model potential $V_{\text {mod }}(r)$ that does include screening but neglects the influence of the magnetic field. We calculate $V_{\text {mod }}$ in the same way as $V(r)$, where, however, we set $B=0$, and therefore instead of the DOS for graphene in a magnetic field, Eq. (4), we use a linear DOS describing graphene for the case of $B=0$ [28],

$$
\rho^{e / h}(E)=\frac{g|E|}{2 \pi \hbar^{2} v_{F}^{2}} .
$$

In the previous studies, the model potential $V_{\text {mod }}(r)$ was chosen ad hoc utilizing various shapes including steplike [23], parabolic [19], power-law [12], and Gaussian [18].

\section{Eigenenergies}

xIn this subsection, we describe the calculation of eigenenergies of electrons in graphene obeying the Dirac equation,

$$
H \Psi(r, \theta)=E \Psi(r, \theta),
$$

with the Hamiltonian Eq. (1).

First of all, note that operator $H$ commutes with the angular momentum operator such that they have a common set of eigenfunctions,

$$
\Psi(r, \theta)=\left(\begin{array}{c}
\chi_{1}(r) e^{i(m-1) \theta} \\
\chi_{2}(r) e^{i m \theta}
\end{array}\right),
$$

with $m=0, \pm 1, \ldots$ being the angular momentum quantum number. Inserting these eigenfunctions into Eq. (11), we obtain a system of equations for the components $\chi_{1}(r), \chi_{2}(r)$,

$$
\begin{aligned}
\frac{V}{\gamma} \chi_{1}-i \frac{d \chi_{2}}{d r}-i \frac{m}{r} \chi_{2}-i \frac{e}{\hbar} A_{\theta} \chi_{2} & =\frac{E}{\gamma} \chi_{1}, \\
-i \frac{d \chi_{1}}{d r}+i \frac{m-1}{r} \chi_{1}+i \frac{e}{\hbar} A_{\theta} \chi_{1}+\frac{V}{\gamma} \chi_{2} & =\frac{E}{\gamma} \chi_{2},
\end{aligned}
$$

where for the perpendicular magnetic field $A_{\theta}=\frac{B r}{2}, A_{r}=0$, as $B \hat{z}=\frac{1}{r}\left(\frac{\partial\left(r A_{\theta}\right)}{\partial r}-\frac{\partial A_{r}}{\partial \theta}\right) \hat{z}$. Note that the operator $H$ is selfadjoint such that it has real eigenenergies and $E\left(m, A_{\theta}\right)=$ $E\left(1-m,-A_{\theta}\right)$ [the latter holds because the change $m \rightarrow(1-$ $m)$ and $A_{\theta} \rightarrow\left(-A_{\theta}\right)$ does not change the system Eq. (13)]. These properties of the operator $H$ have to be preserved in the numerical method. That is why we discretize our domain $[0, R]$ and use a backward-forward finite-difference method with the following properties for $m \geqq 0$ :

(i) One component of the wave function is set to zero at the border of the computational domain $r=R, \chi_{1}(R)=0$.

(ii) The second component of the wave function is set to zero at the origin, $\chi_{2}(0)=0$.

In this way, the system Eq. (13) can be written in matrix form,

$$
M\left(\begin{array}{l}
\chi_{1} \\
\chi_{2}
\end{array}\right)=E\left(\begin{array}{l}
\chi_{1} \\
\chi_{2}
\end{array}\right)
$$

where the explicit expression for matrix $M$ is given by Eq. (14) in Appendix. Matrix $M$ has the properties required by the operator $H$, namely (i) it has real eigenenergies, and (ii) the substitution $m \rightarrow(1-m)$ and $A_{\theta} \rightarrow\left(-A_{\theta}\right)$ does not change the system (14). The former holds as there exists a similarity transformation $M \rightarrow T^{-1} M T$, with $T$ being a diagonal matrix (presented in Appendix), which converts 
matrix $M$ into a self-adjoint matrix (Appendix). Then the energies for negative $m$ can be calculated from the relation $E\left(m, A_{\theta}\right)=E\left(1-m,-A_{\theta}\right)$.

\section{RESULTS AND DISCUSSION}

We consider a graphene sheet of $600 \mathrm{~nm}$ radius on a substrate of thickness $d=10 \mathrm{~nm}$. The external potential $V_{\text {ext }}$ is of $V_{0}=10 \mathrm{~V}$ and $l_{0}=75 \mathrm{~nm}$ (if not stated otherwise). We choose high magnetic fields in the range of 50-80 T. Such a high value of the magnetic field allows us to use a relatively small dot in order to reduce the computational time and still guarantee the presence of the wide compressible strips in the electron density. [Note that the width of the compressible strips is related to the magnetic length $l_{B}=\sqrt{h /|e| B}$ ), such that increasing the dot size twice (to $2 l_{0}$ ) allows one to decrease the magnetic field four times].

Let us now include screening of the external potential by the electron-electron interaction. Consider screening arising from electrons introduced by a gate voltage $e V_{g}=1 \mathrm{eV}$ for different magnetic fields. The total self-consistent potential $V(r)$ for $75 \mathrm{~T}$ is shown in Fig. 2(b) by a bold red line. For comparison, a self-consistent model potential $V_{\text {mod }}(r)$ corresponding to $B=0$ is also indicated (blue line). The screened potential $V(r)$ and the model potential $V_{\text {mod }}(r)$ are qualitatively different: the former shows characteristic plateaus while the latter does not. The plateaus correspond to the compressible strips, and the steep slopes between them correspond to the incompressible strips [24]. Compressible strips are formed in the regions where the Landau levels [thin red curves with $V_{\text {mod }}+\hbar \omega_{C} \sqrt{n}$ in Fig. 2(b)] are partially occupied, that is, they pin or cross the Fermi energy. Due to this partial filling (which occurs in the energy interval

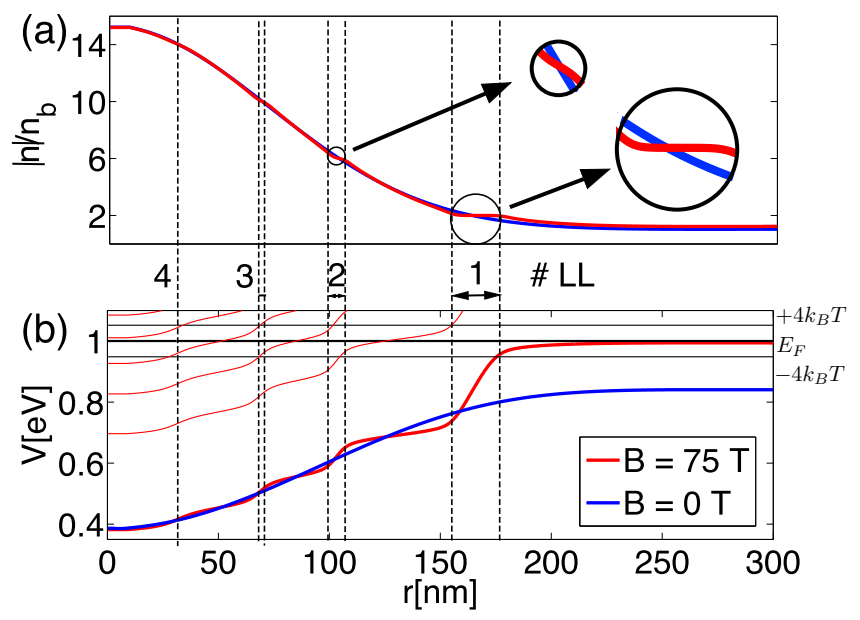

FIG. 2. (Color online) (a) Electrons density $n$ vs distance $r ; n$ is given in units of $n_{b}=\frac{1}{2 \pi l_{b}^{2}}$, the number of states in each Landau level (LL) per unit area. Note that $g=2$ for the zeroth LL and $g=4$ otherwise; (b) potential profiles: the total self-consistent screened potential $V(r)$ and the model potential $V_{\bmod }(r)$ (bold red and blue lines, respectively). Parameters of the external potential $V_{\text {ext }}$ : $V_{0}=10 \mathrm{~V}, l_{0}=75 \mathrm{~nm} . E_{F}=e V_{g}=1 \mathrm{eV}$ (a window $E_{F} \pm 4 k_{B} T$ around $E_{F}$ is indicated). Magnetic field $B=75 \mathrm{~T}$. Thin red lines show $V(r)+\hbar \omega_{C} \sqrt{n}$ with $n=1,2,3,4$, and 5 . The number of occupied Landau levels (\#LL) is indicated in the figure. (a)

(b)

(c)

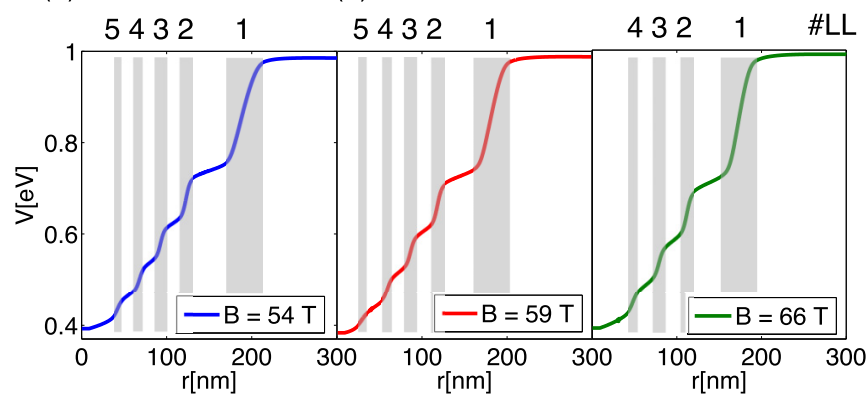

FIG. 3. (Color online) Potential profiles with a maximum of five Landau levels occupied for (a) $B=54 \mathrm{~T}$ and (b) $B=59 \mathrm{~T}$, and with maximally four Landau levels occupied for (c) $B=66 \mathrm{~T}$. Calculated from $V_{\text {ext }}$ with $V_{0}=10 \mathrm{~V}, l_{0}=75 \mathrm{~nm}$, and $e V g=1 \mathrm{eV}$. Regions with fully occupied Landau levels (incompressible strips) are indicated by gray strips and the number of occupied Landau levels.

$\left.E_{F} \pm 4 k_{B} T\right)$, electrons can move freely, like in a metal, and as the name suggests they can compress/redistribute themselves, resulting in the flat completely screened potential and a corresponding varying electron density distribution [Fig. 2(a)]. On the other hand, in the incompressible strips, Landau levels are fully occupied [the number of occupied Landau levels is indicated in Fig. 2(b)] so there is no screening in the potential and the electron density is constant. The positions and sizes of the compressible strips change gradually with the increase of the magnetic field. Furthermore, the number of compressible strips present in the dot is directly related to the number of fully occupied Landau levels; see Figs. 2 and 3. Note that self-consistent calculations of the potential were performed with the lowest possible temperature introduced in order to obtain a numerical convergence. A nonzero temperature smoothes otherwise sharp potential steps (Figs. 2 and 3), still preserving their plateaulike character.

Let us now discuss how the electron-electron interaction affects the eigenenergies of the electrons confined to the quantum dot. Figure 4 shows the eigenenergies as a function of the magnetic-field strength. The presented results are solutions to the coupled system of Eqs. (13) for the angular momentum $m=1$. The blue dots mark eigenenergies of electrons for the model potential $V_{\text {mod }}(r)$ (blue line in Fig. 2), while the red dots correspond to the total screened potential $V(r)$ (calculated self-consistently for different $B$ ).

We first note that the calculated eigenenergies closely follow the pure Landau levels $E_{n}=\hbar \omega_{C} \sqrt{n}+U_{0}$ (with $U_{0}=$ $386.99 \mathrm{meV}$ ) depicted by green curves in Fig. 4 (that is why we will refer to the eigenenergies of the system at hand in the considered magnetic-field interval as the Landau levels as well). The shift $U_{0}$ in pure Landau levels is a consequence of a nonzero gate voltage $e V_{g}$, which moves the charge neutrality point upward. Second, note that for angular momentum $m$, the only Landau levels present are those with indices $|n| \geqslant m$. Without loss of generality, let us continue with the case $m=1$ (Fig. 4).

Let us now discuss the most important feature of the eigenenergies, namely the plateaus present for the case of the screened total potential $V(r)$ and absent for the case of the model potential $V_{\text {mod }}(r)$. [The plateau regions are indicated 


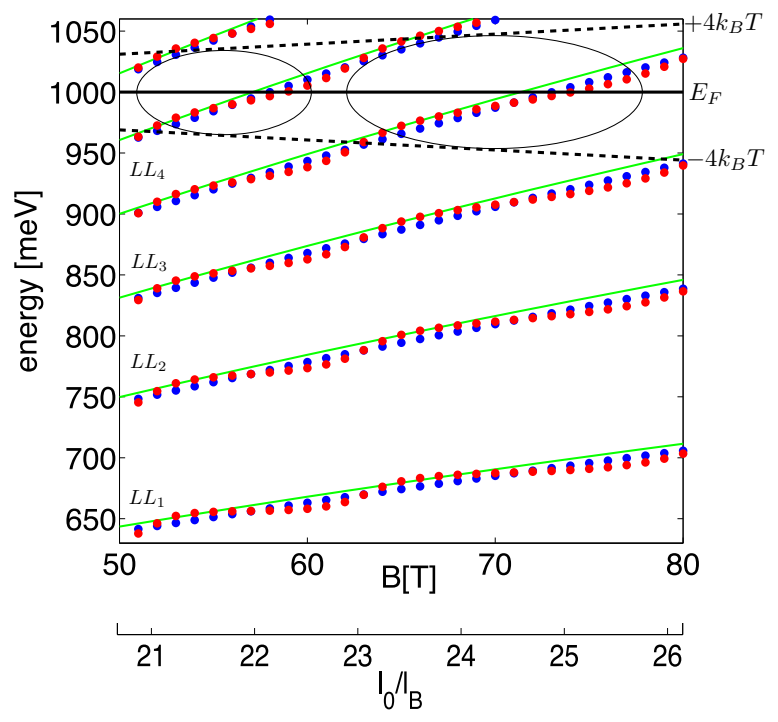

FIG. 4. (Color online) Eigenenergies vs magnetic field. Calculations are done for the total screened potential $V(r)$ (blue dots) and for the model potential $V_{\text {mod }}(r)$ with the Fermi energy $E_{F}=e V_{g}=1 \mathrm{eV}$. Green lines indicate pure Landau levels $L L_{n}$ with the energies $E_{n}=\hbar \omega_{C} \sqrt{n}+U_{0} ; U_{0}=386.99 \mathrm{meV}$. Ellipses indicate plateaus formed due to the pinning of the eigenstates belonging to the partially filled states to the Fermi energy. The window $E_{F} \pm 4 k_{B} T$ corresponding to the states that are partially filled is indicated. Note that a temperature $T$ introduced to achieve a convergence of numerical calculations increases with $B$. The magnetic field is indicated both in units of $\mathrm{T}$ and as a ratio $l_{0} / l_{B}$.

by ellipses around the Fermi energy level in Fig. 4; a closer look at the eigenenergies corresponding to the Landau levels $n=1$ and $n=-1$ (i.e., $L L_{1}$ and $L L_{-1}$ ) is given in Fig. 5; note that plateaus are smeared in the window $E_{F} \pm 4 k_{B} T$ by the effect of a finite temperature]. These plateaus are related to the steplike structure of the corresponding potential $V(r)$. Indeed, consider, e.g., a magnetic field $B=54 \mathrm{~T}$ corresponding to the case of four fully occupied Landau levels in the dot and

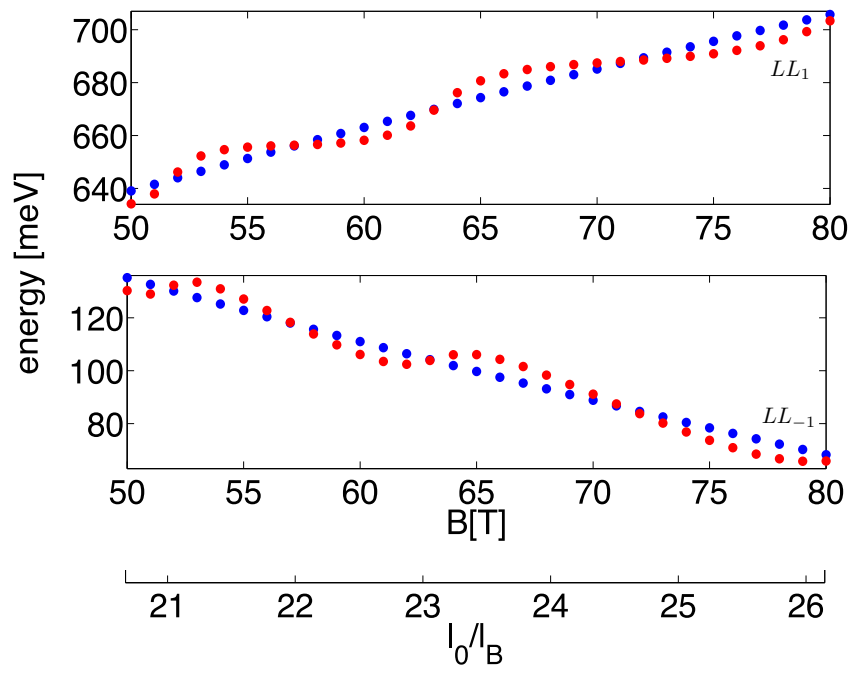

FIG. 5. (Color online) The same as in Fig. 4 but showing the eigenstates corresponding to $L L_{1}$ and $L L_{-1}$.

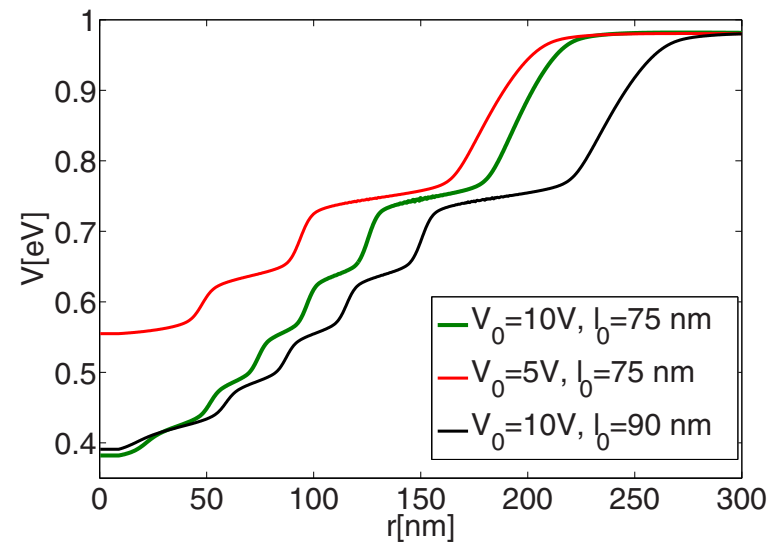

FIG. 6. (Color online) (a) Potential profiles for fixed magnetic field $B=50 \mathrm{~T}$, gate voltage $e V_{g}=1 \mathrm{eV}$, and different dot parameters.

one partially filled $\left(L L_{5}\right)$ that is pinned to the Fermi energy; see Fig. 3. Increasing the magnetic field to $B=59 \mathrm{~T}$ does not change the number of occupied Landau levels, and the potential remains practically unchanged because electrons in $L L_{5}$ are easily redistributed to screen the external changes. As a result, the position of the eigenenergies does not change much when the magnetic field is varied from 54 to $59 \mathrm{~T}$ (i.e., the eigenenergies exhibit a plateau in this interval, as indicated by a small ellipse in Fig. 4). However, when the magnetic field is further increased to $B=66 \mathrm{~T}$, the potential changes because the number of fully occupied Landau levels is reduced to three, and it is now eigenstates corresponding to $L L_{4}$ that are pinned to $E_{F}$ (large ellipse in Fig. 4). As a result, the eigenenergies exhibit a plateaulike structure in their magnetic-field dependence because of the consecutive pinning to $E_{F}$ of the eigenstates belonging to the highest partially occupied Landau level as a given $B$.

Due to the rigidity of the density of states, which keeps the distance between the adjacent Landau levels fixed [see Eq. (4)], similar plateaus are inherited by all other electron Landau levels $(n>0)$. Note that the same rigidity introduces steep slopes in the hole Landau levels $(n<0)$ (Fig. 5) for the magnetic fields when the electron Landau levels exhibit plateaus.

Finally, we briefly discuss how the dot parameters, namely the depth $V_{0}$ and extension $l_{0}$, influence the energy plateaus. Figure 6 shows that the widths of the energy plateaus increase when $V_{0}$ decreases or $l_{0}$ increases (i.e., when the dot becomes shallower or less steep). This is an expected behavior. Indeed, both a decrease of $V_{0}$ and an increase of $l_{0}$ make the dot potential less steep. Therefore, electrons can screen the external potential more easily, which leads to the increase of the width of the compressible strips in the potential and density, and, as a result, to wider plateaus in the energy spectrum.

\section{EXPERIMENT DESIGN}

In this section, we discuss experimental measurements that can be conducted to verify the results presented in this article. The most direct way to measure the surface potential is to use a Kelvin probe force microscope (KPFM) [40]. The 
KPFM is based on the atomic force microscope (AFM) and measures the contact potential difference, i.e., the difference between the work functions of the AFM tip and the contact area of a sample. It can work in two different modes: (i) amplitude-modulated (AM), where the tip of the microscope is in contact with the sample and is deflected by the repulsive tip-sample force, and (ii) frequency-modulated (FM), which is a noncontact mode in which the tip oscillates at the resonant frequency, which changes due to the interaction with the sample. Recently, the KPFM was tested on graphene structures [41-44]. In particular, the experiments showed that the KPFM-AM has better contact potential resolution than KPFM-FM. However, its spatial resolution is smaller that that for the KPFM-FM (50-70 nm versus $20 \mathrm{~nm}$ ) [41]. Moreover, it was shown recently that the KPFM can detect step changes in the surface potential due to the change in the number of graphene layers [42,44] (as the number of layers is related to the interlayer screening effect [44]) or appearance of wrinkles [43]. Therefore, we believe that this high accuracy device should be able to detect steplike potential profiles (Figs. 2, 3, and 6), even in small quantum dots.

Finally, we note that the investigation of the optical transitions between the levels represents a powerful tool to probe the spectrum of quantum dots. For the graphene islands and for the field-induced dots with a parabolic potential for low magnetic fields, such transitions were considered in $[19,45,46]$. In our case of a strong magnetic field, the experimental study of the optical transitions would not detect the presence of energy plateaus. Indeed, for high magnetic fields, optical transitions are possible for a fixed angular momentum $m$, and, as there is no mixing between the Landau levels, the transitions should be the strongest between the adjacent Landau levels [19]. Then, even though the energy levels for the self-consistent screened potential $V(r)$ are changed as compared to those for the model potential $V_{\text {mod }}(r)$, where the formation of the compressible strips is not accounted for, the energy difference between the levels (manifested in the optical spectra) remains the same in both cases.

\section{CONCLUSIONS}

In this paper, we investigated the electronic properties of graphene quantum dots defined by a smooth potential and a high perpendicular magnetic field. Our principal goal was to investigate how the electron-electron interaction affects the energies of electrons trapped in the quantum dots. The electron-electron interaction was treated in the Thomas-Fermi approximation and was included in the model via a screened potential calculated self-consistently. The electron dynamics in the dot was described by a continuous Dirac Hamiltonian. To obtain electron energies, we used a numerical backwardforward finite-difference method. Our results show that electron-electron interaction introduces compressible strips in the quantum dots that manifested themselves in the steps in the self-consistent total potential. As a consequence, electrons energies are modified leading to the appearance of energy plateaus as a function of the magnetic field. The width of the energy plateaus increases when the confinement becomes shallower and less steep. Finally, we discussed how the results presented in our paper can be verified using the Kelvin probe force microscope measurements.

It is well known that the formation and charging of the compressible strips in and around electrostatically defined dots and antidots in conventional semiconductor heterostructures lead to many interesting phenomena in the edge-state transport regime. We therefore believe that the results presented in our study revealing the character of the confining potential and the structure of the energy levels can be important for designing and understanding the transport experiments in the confined geometry for graphene quantum dots and antidots.

\section{ACKNOWLEDGMENTS}

The authors thank V. Kozlov for the discussion on the mathematical aspects of the paper, and F. Berntsson and A. V. Volkov for help with the programming codes. The Center for Nanostructured Graphene (CNG) is sponsored by the Danish National Research Foundation (DNRF58).

\section{APPENDIX: TRANSFORMATION MATRIX}

Let us introduce discretization of the radial component $r$ with discretization step $h$. From the backward-forward finite-difference method for $m>0$ and the boundary conditions described in Sec. IIC, we get the following problem for the eigenenergy $E$ :

$$
M\left(\begin{array}{c}
\chi_{1}(h) \\
\chi_{1}(2 h) \\
\chi_{1}(3 h) \\
\vdots \\
\chi_{2}(h) \\
\chi_{2}(2 h) \\
\chi_{2}(3 h) \\
\vdots
\end{array}\right)=\frac{E}{\gamma}\left(\begin{array}{c}
\chi_{1}(h) \\
\chi_{1}(2 h) \\
\chi_{1}(3 h) \\
\vdots \\
\chi_{2}(h) \\
\chi_{2}(2 h) \\
\chi_{2}(3 h) \\
\vdots
\end{array}\right)
$$


with

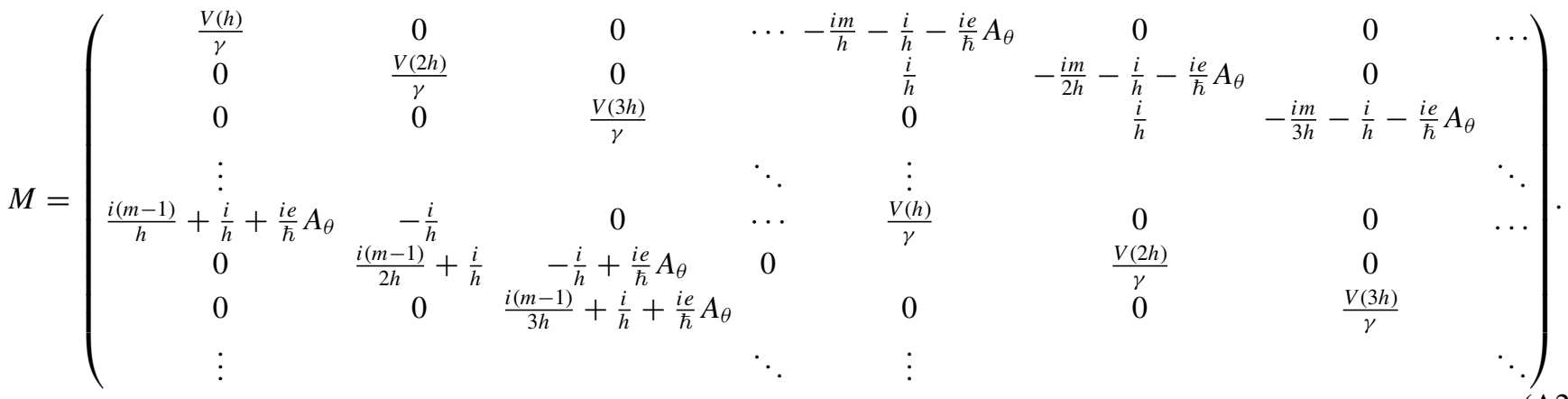

Matrix $T$ used in the similarity transformation of matrix $M$ has the following diagonal form:

$$
T=\left(\begin{array}{cccccccc}
1 & 0 & 0 & \cdots & 0 & 0 & 0 & \cdots \\
0 & \frac{\sqrt{B h^{2}+m}}{\sqrt{B h^{2}+m+1}} & 0 & & 0 & 0 & 0 & \\
0 & 0 & \frac{\sqrt{B h^{2}+m} \sqrt{4 B h^{2}+m+1}}{\sqrt{B h^{2}+m+1} \sqrt{4 B h^{2}+m+2}} & 0 & 0 & 0 & \\
\vdots & & & \ddots & \vdots & & & \\
0 & 0 & 0 & \cdots & \frac{\sqrt{B h^{2}+m}}{\sqrt{B h^{2}+m+1}} & 0 & 0 & \ddots \\
0 & 0 & 0 & 0 & & \frac{\sqrt{B h^{2}+m} \sqrt{4 B h^{2}+m+1}}{\sqrt{B h^{2}+m+1} \sqrt{4 B h^{2}+m+2}} & 0 & \\
0 & 0 & 0 & & 0 & 0 & \frac{\sqrt{B h^{2}+m} \sqrt{4 B h^{2}+m+1} \sqrt{9 B h^{2}+m+2}}{\sqrt{B h^{2}+m+1} \sqrt{4 B h^{2}+m+2} \sqrt{9 h^{2}+m+3}} & \\
\vdots & & & \ddots & \vdots & & & \ddots
\end{array}\right) .
$$

[1] M. Reimann and M. Manninen, Rev. Mod. Phys. 74, 1283 (2002).

[2] D. Heitmann and J. Kotthaus, Phys. Today 46(6), 56 (1993).

[3] J. A. Smyder and T. D. Krauss, Mater. Today 14, 382 (2011).

[4] K. S. Novoselov, A. K. Geim, S. V. Morozov, D. Jiang, Y. Zhang, S. V. Dubonos, I. V. Grigorieva, and A. A. Firsov, Science 306, 666 (2004).

[5] A. V. Rozhkov, G. Giavaras, Y. P. Bliokh, V. Freilikher, and F. Nori, Phys. Rep. 503, 77 (2011).

[6] K. A. Ritter and J. W. Lyding, Nat. Mater. 8, 235 (2009).

[7] S. Engels, A. Epping, C. Volk, S. Korte, B. Voigtlander, K. Watanabe, T. Taniguchi, S. Trellenkamp, and C. Stampfer, Appl. Phys. Lett. 103, 073113 (2013).

[8] S. Schnez, F. Molitor, C. Stampfer, J. Güttinger, I. Shorubalko, T. Ihn, and K. Ensslin, Appl. Phys. Lett. 94, 012107 (2009).

[9] A. Barreiro, H. S. J. van der Zant, and L. M. K. Vandersypen, Nano Lett. 12, 6096 (2012).

[10] A. D. Güçlü, P. Potasz, M. Korkusinski, and P. Hawrylak, Graphene Quantum Dots (Springer, Berlin, Heidelberg, 2014).

[11] A. D. Güçlü, P. Potasz, O. Voznyy, M. Korkusinski, and P. Hawrylak, Phys. Rev. Lett. 103, 246805 (2009).

[12] G. Giavaras, P. A. Maksym, and M. Roy, J. Phys.: Condens. Matter 21, 102201 (2009).

[13] S. Moriyama, Y. Morita, E. Watanabe, and D. Tsuya, Appl. Phys. Lett. 104, 053108 (2014).

[14] J. Zhao-Tan, Y. Cheng-Long, and D. Quan-Li, Chin. Phys. B 21, 027303 (2012).

[15] M. I. Katsnelson, K. S. Novoselov, and A. K. Geim, Nat. Phys. 2, 620 (2006).
[16] P. A. Maksym, M. Roy, M. F. Craciun, S. Russo, M. Yamamoto, S. Tarucha, and H. Aoki, J. Phys.: Conf. Ser. 245, 012030 (2010).

[17] G. Giavaras and F. Nori, Phys. Rev. B 85, 165446 (2012).

[18] P. A. Maksym and H. Aoki, Phys. Rev. B 88, 081406(R) (2013).

[19] S. C. Kim, J. W. Lee, and S.-R. Eric Yang, J. Phys.: Condens. Matter 24, 495302 (2012).

[20] P. S. Park, S. C. Kim, and S.-R. Eric Yang, Phys. Rev. B 84, 085405 (2011).

[21] O. V. Gamayun, E. V. Gorbar, and V. P. Gusynin, Phys. Rev. B 83, 235104 (2011).

[22] S. Schnez, K. Ensslin, M. Sigrist, and T. Ihn, Phys. Rev. B 78 , 195427 (2008).

[23] A. Matulis and F. M. Peeters, Phys. Rev. B 77, 115423 (2008).

[24] D. B. Chklovskii, B. I. Shklovskii, and L. I. Glazman, Phys. Rev. B 46, 4026 (1992).

[25] S. Ihnatsenka and I. V. Zozoulenko, Phys. Rev. B 74, 201303(R) (2006).

[26] S. Ihnatsenka, I. V. Zozoulenko, and G. Kirczenow, Phys. Rev. B 80, 115303 (2009).

[27] S. Ihnatsenka and I. V. Zozoulenko, Phys. Rev. B 77, 235304 (2008).

[28] N. M. R. Peres, J. N. B. Rodrigues, T. Stauber, and J. M. B. Lopes dos Santos, J. Phys.: Condens. Matter 21, 344202 (2009).

[29] W. Häusler and R. Egger, Phys. Rev. B 80, 161402(R) (2009).

[30] M. Kataoka, C. J. B. Ford, G. Faini, D. Mailly, M. Y. Simmons, D. R. Mace, C.-T. Liang, and D. A. Ritchie, Phys. Rev. Lett. 83, 160 (1999).

[31] F. E. Camino, W. Zhou, and V. J. Goldman, Phys. Rev. B 76, 155305 (2007). 
[32] M. Kataoka and C. J. B. Ford, Phys. Rev. Lett. 92, 199703 (2004).

[33] I. Karakurt, V. J. Goldman, J. Liu, and A. Zaslavsky, Phys. Rev. Lett. 87, 146801 (2001).

[34] S. Ihnatsenka and I. V. Zozoulenko, Phys. Rev. Lett. 99, 166801 (2007).

[35] T. Andrijauskas, A. Shylau, and I. Zozoulenko, Lith. J. Phys. 52, 63 (2012).

[36] A. H. Castro Neto, F. Guinea, N. M. R. Peres, K. S. Novoselov, and A. K. Geim, Rev. Mod. Phys. 81, 109 (2009).

[37] K. Lier and R. R. Gerhardts, Phys. Rev. B 50, 7757 (1994).

[38] J. H. Oh and R. R. Gerhardts, Phys. Rev. B 56, 13519 (1997).

[39] A. V. Volkov, A. A. Shylau, and I. V. Zozoulenko, Phys. Rev. B 86, 155440 (2012).
[40] W. Melitz, J. Shen, A. C. Kummel, and S. Lee, Surf. Sci. Rep. 66, 1 (2011).

[41] V. Panchal, R. Pearce, R. Yakimova, A. Tzalenchuk, and O. Kazakova, Sci. Rep. 3, 2597 (2013).

[42] C. Held, T. Seyller, and R. Bennewitz, Beilstein J. Nanotechnol. 3, 179 (2012)

[43] S. Ladaka, J. M. Balla, D. Moseleya, G. Edab, W. R. Branforda, M. Chhowallab, T. D. Anthopoulosa, and L. F. Cohena, Carbon 64, 35 (2013).

[44] N. J. Lee, J. W. Yoo, Y. J. Choi, C. J. Kang, D. Y. Jeon, D. C. Kim, S. Seo, and H. J. Chung, Appl. Phys. Lett. 95, 222107 (2009).

[45] M. Grujić, M. Zarenia, M. Tradić, and F. M. Peeters, Phys. Scr. T 149, 014056 (2012).

[46] H.-Y. Chen, V. Apalkov, and T. Chakraborty, Phys. Rev. Lett. 98, 186803 (2007). 